\title{
Synthesis of Stereodefined 1,1-Diborylalkenes via Copper-Catalyzed Diboration of Terminal Alkynes
}

\author{
Yang Gao, Zhong-Qian Wu, Keary M. Engle* \\ Department of Chemistry, The Scripps Research Institute, 10550 North Torrey Pines Road, La Jolla, California 92037, United States
}

Supporting Information Placeholder

\begin{abstract}
A copper-catalyzed method for the E-selective 1,1-diboration of terminal alkynes is described. The tandem process involves sequential dehydrogenative borylation of the alkyne substrate with HBdan (HBdan = 1,8-diaminonaphthalatoborane), followed by hydroboration with HBpin (HBpin = pinacolborane). This method proceeds efficiently under mild conditions, furnishing 1,1-diborylalkenes with excellent stereoselectivity and broad functional-group tolerance. Taking advantage of the different reactivities of the two boryl moieties, the products can then be employed in stepwise cross-couplings with aryl halides for the stereocontrolled construction of trisubstituted alkenes.
\end{abstract}

Alkenyl boronic acids and their derivatives are non-toxic, shelf-stable organometallic compounds that react with high fidelity in a range of $\mathrm{C}-\mathrm{C}$ and $\mathrm{C}$-heteroatom couplings, making them useful reagents in organic synthesis. ${ }^{1} 1,1-D i b o r y l a l k e n e s$ are an emerging subclass that offer exciting potential for accessing multisubstituted olefins in a stereocontrolled manner through sequential reaction at each of the two $\mathrm{C}-\mathrm{B}$ bonds. ${ }^{2}$ To this end, many approaches to synthesize 1,1-diboryl alkenes bearing two -Bpin groups $($ Bpin $=$ pinacolatoboryl $)$ have been developed from alkene $^{2 \mathrm{c}, 3}$ and alkyne starting materials. ${ }^{4}$ When such compounds are then employed in cross-coupling, the inherently similar reactivity of the two C-Bpin bonds makes it challenging to achieve high selectivity for a mono-functionalized product. Indeed, successful mono-selectivity at the less hindered position has only been demonstrated when the 1,1-diborylalkene contains an aryl group at the 2-position and when an aryl iodide is employed as the electrophile (Scheme $1 \mathrm{~A}$ ). ${ }^{2 \mathrm{c}, 4 \mathrm{~b}}$

We reasoned that the scope of substrates and coupling partners could be expanded if the two boron centers were differentially protected with one $-($ pin $)($ pin $=$ pinacolate $)$ and one $-($ dan $)($ dan $=1,8$-diaminonaphthalenyl) group (Scheme 1 A). The Bdan group is well known to possess diminished Lewis acidity, and to be generally inactive toward transmetalation, a key step of cross-coupling. ${ }^{5}$ This so-called protected boron moiety can be reactivated by either deprotection under acidic condition, ${ }^{5}$ or interaction with $\mathrm{KO} t \mathrm{Bu}$ or $\mathrm{Ba}(\mathrm{OH})_{2} \cdot{ }^{6}$ The sequential masking/unmasking strategy enabled by the Bdan group has been successfully applied to iterative cross-coupling, providing efficient and concise approaches to functional organic molecules including complex oligorenes, ${ }^{\text {Sa-c, } 6 \mathrm{c}}$ and optoelectronic materials. ${ }^{5 \mathrm{~d}}$ Moreover, these isomerically pure 1,1-diborylalkenes represent a promising class of prochiral substrates to generate enantioenriched 1,1-diborylalkanes, ${ }^{7}$ that are valuable versatile building blocks to access enantioenriched functionalized alkanes. ${ }^{8}$

In view of the unique synthetic value of differentially protected 1,1-diborylalkenes, practical synthetic methods to access such complexes in a stereodefined manner are desirable. However, few methods are currently available. In 2017, Chirik and colleagues described a Co-catalyzed 1,1-diboration of aliphatic alkynes to synthesize (Z)-1,1-diborylakenes with use of the mixed diboron reagent pinB-Bdan (Scheme $1 \mathrm{~B}){ }^{4 \mathrm{~b}}$ Later, Marder reported a base-catalyzed stereoselective diboration of alkynyl esters and amides with pinB-Bdan (Scheme 1 C). ${ }^{9}$ Both methods, though highly enabling in their own right, have limited substrate scope and only provide access to the $Z$-configured products.

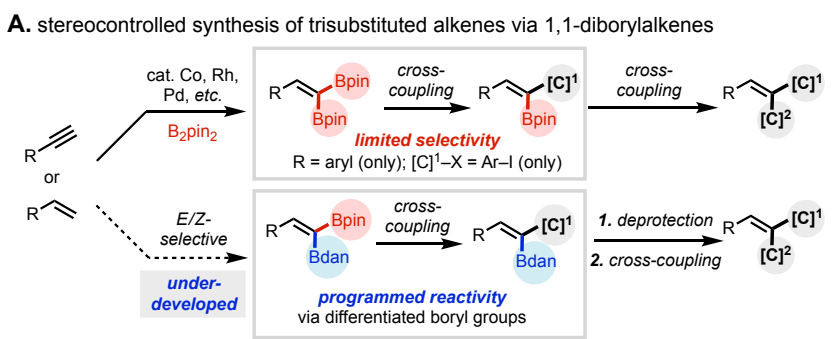

B. Z-selective cobalt-catalyzed two-component approach [Chirik, 2017]

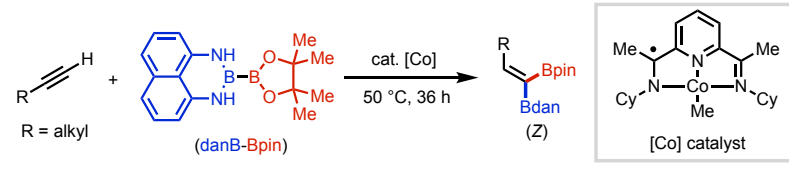

C. Z-selective base-catalyzed two-component approach [Marder, 2020]

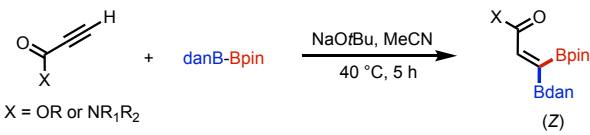

D. E-selective copper-catalyzed three-component approach (this work)

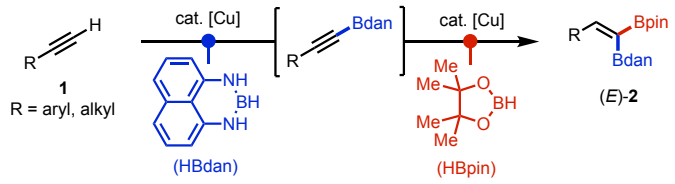

Scheme 1. (A) Synthesis and reactivity of 1,1-diborylalkenes. (B), (C) Prior art to access Z-configured 1,1-diborylalkenes. (D) This work.

Driven by our interest in developing new metal-catalyzed alkyne difunctionalization methods, we recently reported a $\mathrm{CuH}$-catalyzed cascade process to access enantioenriched $\alpha$-aminoboron compounds via sequential hydroboration and hydroamination of terminal alkynes. ${ }^{10}$ Here we report an exclusively $E$-selective $\mathrm{Cu}$-catalyzed three-component reaction to produce 1,1-diborylakenes through a tandem sequence comprised of dehydrogenative $\mathrm{C}(\mathrm{sp})-\mathrm{H}$ borylation with HBdan and hydroboration of the resulting alkynylBdan intermediate with HBpin (Scheme 1 D).

Our investigation commenced by examining reaction conditions using phenylacetylene (1a) as pilot substrate, with HBdan and HBpin as coupling partners. After extensive optimization, we identified an effective protocol in which HBdan is first mixed with $1 \mathrm{a}$ in the presence of $5 \mathrm{~mol} \%$ $\mathrm{Cu}(\mathrm{OAc})_{2}$ and $5 \mathrm{~mol} \%\left(R_{a}\right)$-DTBM-SEGPHOS in THF for $15 \mathrm{~min}$. After this period HBpin is added, and the reaction is allowed to stir for an additional $16 \mathrm{~h}$ at room temperature, at which point the desired product 2a is isolated in $69 \%$ yield. (Table 1, entry 1 ) The timing of HBpin addition was found to be important. The yield of $\mathbf{2 a}$ decreased when HBpin added earlier, with larger amount of side products observed (Table 1, entries 2-3). 
Another key finding from these studies was that acetate counteranion and $(R a)$-DTBM-SEGPHOS ligand were both required (Table 1, entries 4-12). CuOAc was slightly less effective than $\mathrm{Cu}(\mathrm{OAc})_{2}$ (Table 1, entry 4), while other copper sources such as $\mathrm{CuBr}$ and $\mathrm{CuCl}$, together with $\mathrm{NaO} t \mathrm{Bu}$, showed no catalytic activity. In terms of other ligands tested, $\left(R_{a}\right)$-DM-SEGPHOS gave slightly lower yield of $\mathbf{2 a}$ than $\left(R_{a}\right)$ DTBM-SEGPHOS (Table 1, entry 12). Other phosphine ligands unfortunately led to either low yield or no reaction. There was no reaction in the absence of a ligand (Table 1, entry 13). The structure and (E)-stereochemical configuration of 2a were unambiguously assigned by X-ray crystallography (Table 2, top left).

Table 1. Optimization of reaction conditions for $\mathrm{Cu}$-catalyzed 1,1-diboration of phenylacetylene.

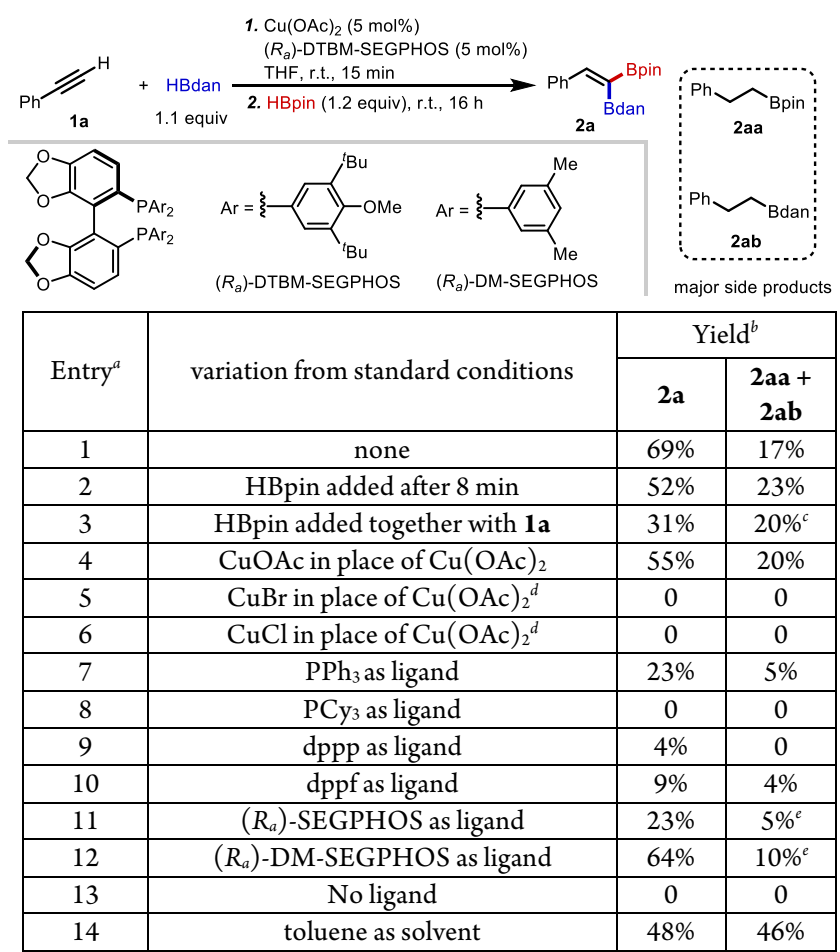

a Standard reaction conditions: $1 \mathrm{a}(0.10 \mathrm{mmol})$, HBdan $(0.11 \mathrm{mmol})$, HBpin (0.12 mmol), $\mathrm{Cu}(\mathrm{OAc})_{2}(5 \mathrm{~mol} \%)$, and $\left(R_{a}\right)$-DTBMSEGPHOS (5 mol\%) in THF $(0.25 \mathrm{~mL}) .{ }^{1}{ }^{1} \mathrm{H}$ NMR yield using $\mathrm{CH}_{2} \mathrm{Br}_{2}$ as internal standard. ${ }^{c} 10 \%$ of $\mathrm{PhCHCH}\left(\right.$ Bpin) was also observed. ${ }^{d}$ Together with $\mathrm{NaO} t \mathrm{Bu}(10 \%) .{ }^{e} 9 \%$ of $\mathrm{PhCHC}(\mathrm{Bpin})_{2}$ was observed.

Next, we evaluated the scope and functional group compatibility of this stereoselective process (Table 2). The reactions with aryl-substituted alkynes were found sensitive to both electronic and steric effects. Aryl acetylenes with electron-donating groups normally performed better than those with electron-withdrawing groups. For example, $p-\mathrm{MeO}$-substituted phenylacetylene gave product $2 \mathbf{c}$ in $70 \%$ yield, while $p-\mathrm{CF}_{3}-$ substituted phenylacetylene gave product $2 \mathrm{f}$ in $47 \%$ yield. Diboration of para-substituted phenylacetylenes generally occurred smoothly while meta- and ortho-substituted phenylacetylenes gave relatively low yield. A range of functional groups, such as halides $(\mathbf{2 e}, \mathbf{2 k}, \mathbf{2 l}, \mathbf{2 m})$, an ester $(\mathbf{2 g})$, ethers $(\mathbf{2} \mathbf{c}, \mathbf{2} \mathbf{i})$ and amines $(\mathbf{2 d}, \mathbf{2} \mathbf{j})$, were well tolerated. 2-Ethynylnaphthalene and 3-ethynylthiophene underwent diboration as well to give corresponding 1,1-diborylalkenes (2h, 2n).

1,1-Diboration was similarly effective with aliphatic alkynes, furnishing the corresponding E-configured products as well. The structure of 1,1diborylalkene 20 was confirmed by X-ray crystallography (Table 2, bottom left). Remarkably, 1-ethynylcyclohexene underwent 1,1-diboration to furnish product $2 \mathbf{s}$ with $\mathrm{C}=\mathrm{C}$ double bond intact. Functional groups like carbonate (2p), ether $(\mathbf{2 q})$ and silyl ether $(\mathbf{2 t})$ were tolerated with products isolated in useful yields.
Table 2. Copper-catalyzed stereoselective 1,1-diboration of terminal alkynes. $^{a}$

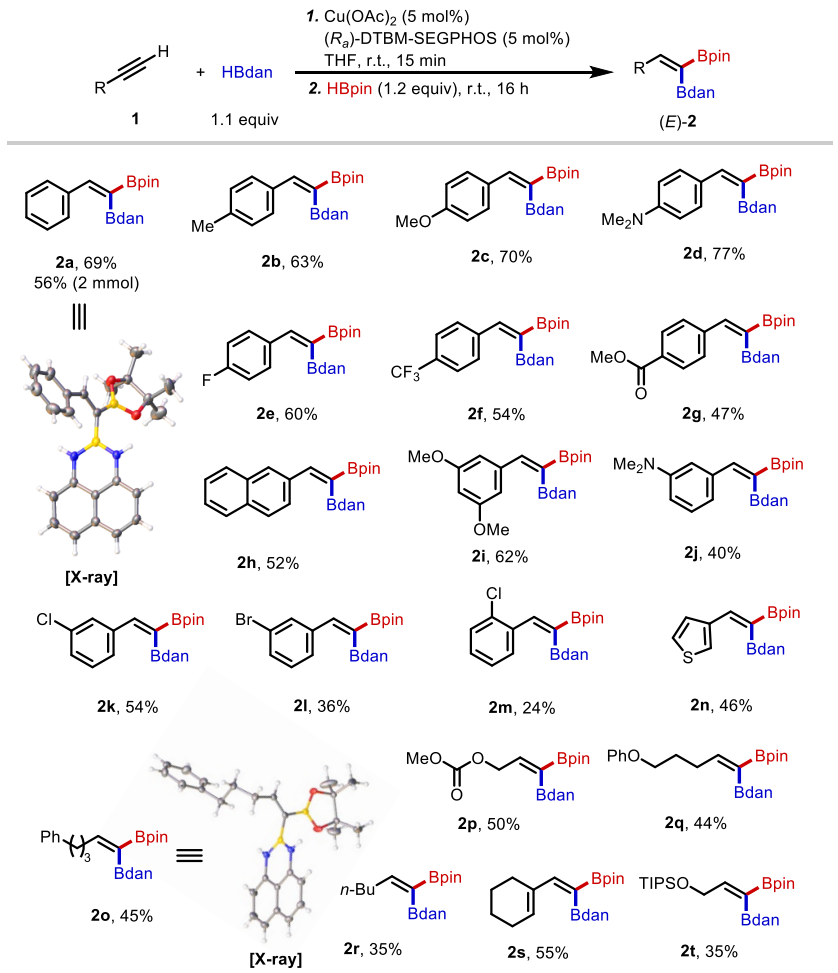

${ }^{a}$ Conditions: $0.10 \mathrm{mmol}$ scale, THF $(0.25 \mathrm{~mL})$. Percentages correspond to isolated yields.

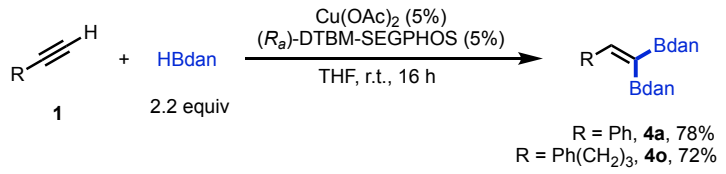

Interestingly, when terminal alkynes 1 were reacted with 2.2 equiv HBdan in the absence of HBpin, 1,1-homodiboration proceeded smoothly, furnishing 1,1-diborylalkenes $\mathbf{4}$ bearing two -Bdan groups in excellent yields (eq. 1).

To illustrate the practical utility of this procedure, we performed stepwise Suzuki-Miyaura cross-couplings of 1,1-diborylalkenes (Scheme 2). The selective monoarylation of $\mathbf{2 a}$ worked well using 4-iodotoluene as coupling partner under standard cross-coupling conditions, giving $92 \% 5 \mathrm{a}$ after $12 \mathrm{~h}$ at $30^{\circ} \mathrm{C}$. Notably, the reaction with 4-bromotoluene also proceeded, albeit at a higher temperature of $80^{\circ} \mathrm{C}$, giving product $\mathbf{5 a}$ in $85 \%$ yield with no diarylation product detected. Next, the Bdan group of $\mathbf{5} \mathbf{a}$ was deprotected, and the resulting boronic acid was carried forward without purification in a second cross-coupling reaction with 4 iodoanisole to produce triarylated alkene $\mathbf{6} \mathbf{a}$ as a single stereoisomer.

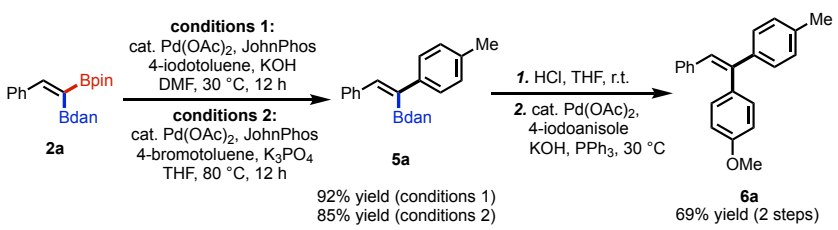

Scheme 2. Stepwise Suzuki-Miyaura coupling reactions of $\mathbf{2 a}$.

Having established the scope and utility of the $(E)$-selective alkyne 1,1diboration method, we shifted our attention to investigating the reaction mechanism. Subjecting sterically bulky mesitylacetylene to the standard reaction conditions did not lead to formation of the typical 1,1-diboryalted product; instead, alkynylBdan $3 \mathbf{u}$ was isolated in $55 \%$ yield (eq. 2). This result suggests that alkynylBdan is the product from first 
step of the tandem process and that the second hydroboration step is sensitive to steric hindrance. Consistent with this notion, the reaction between $\mathbf{2 a}$ and $\mathrm{HBdan}$ before addition of $\mathrm{HBpin}$ was monitored by ${ }^{1} \mathrm{H}$ NMR spectroscopy (eq. 3), and $16 \%$ alkynylBdan $3 \mathbf{a}$ and $\mathrm{H}_{2}$ were both observed after $30 \mathrm{~min}$ (SI, Figure S1). To further support our hypothesis, the proposed intermediate 3a was independently synthesized and submitted to the reaction with HBpin under the standard conditions. Hydroboration of 3a proceeded smoothly to furnish $2 \mathbf{a}$ in $60 \%$ yield (eq. 4).

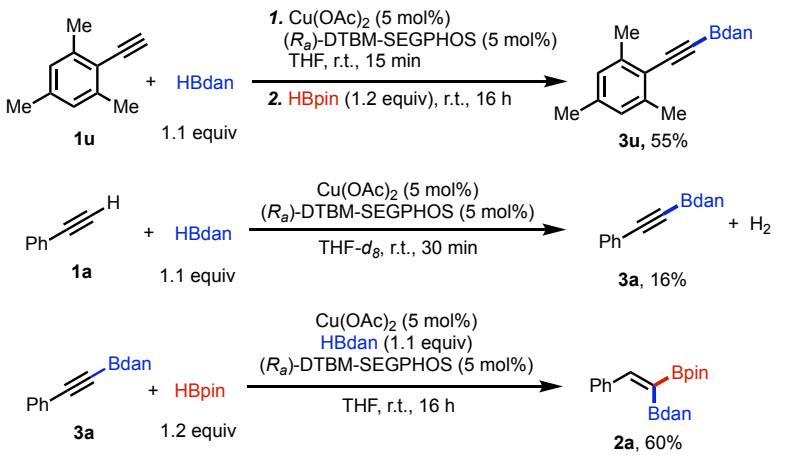

Based on our experimental observations and previous reports, ${ }^{11-13} \mathrm{a} \mathrm{Cu}-$ catalyzed tandem process comprised of dehydrogenative borylation of terminal alkynes and following hydroboration is proposed (Scheme 3). $[\mathrm{LCuOAc}] \mathbf{a 1}$, generated by reduction of $\mathrm{Cu}(\mathrm{OAc})_{2}$ in the presence of phosphine, ${ }^{14}$ reacts with terminal alkynes to give the alkynylcopper intermediate $\mathbf{a} 2$ and HOAc. ${ }^{13 b, 15}$ Following $\sigma$-bond metathesis between $\mathbf{a} 2$ and $\mathrm{HBdan}$ gives alkynylBdan $\mathbf{3}$ and $[\mathrm{LCuH}] \mathbf{a} 3 .{ }^{11,16}$ syn-Insertion of $\mathbf{a} 3$ to intermediate 3 generates alkenyl copper species a4, ${ }^{12,13,17}$ which then undergoes $\sigma$-bond metathesis with HBpin to furnish the $(E)$-1,1-diborylalkenes and regenerate $[\mathrm{LCuH}]$ a3.

Under the optimal conditions, prior to addition of HBpin, the right cycle alone proceeds through several turnovers to build up $\mathbf{3 a}$, and upon addition of HBpin the left cycle turns on. This strategy limits the formation of side products caused by the high reactivity of HBpin. In the 1,1-homodiboration system with $\mathrm{HBdan}$ only, both cycles presumably proceed in parallel. ${ }^{18}$

In conclusion, we have reported the first $(E)$-selective synthesis of 1,1diborylalkenes bearing one -Bpin group and one -Bdan group from terminal alkynes via a $\mathrm{Cu}$-catalyzed tandem process. A wide range of aryland alkyl-substituted alkynes underwent this transformation, giving the corresponding 1,1-diborylalkenes with broad functional group tolerance. We have also demonstrated that differentially protected 1,1-diborylalkenes are useful synthetic intermediates for the construction of multi-substituted alkenes with stereocontrol. Further applications of 1,1-diborylalkenes for the synthesis of more complex compounds are being pursued in our laboratory.

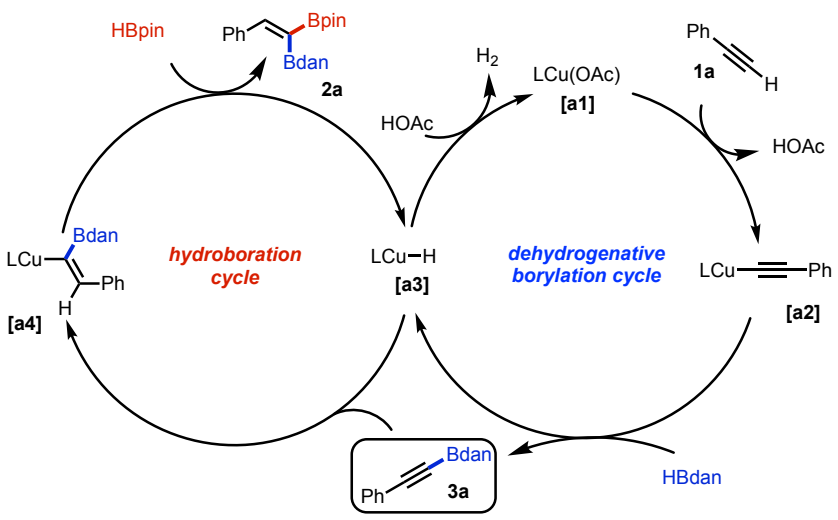

Scheme 3. Proposed mechanism.

\section{ASSOCIATED CONTENT}

\section{Supporting Information}

The Supporting Information is available free of charge at DOI: [filled later].

Detailed experimental procedures and compound characterization (PDF)

Crystallographic data for $\mathbf{2 a}$ (CIF)

Crystallographic data for $\mathbf{2 o}$ (CIF)

\section{AUTHOR INFORMATION}

\section{Corresponding Author}

*E-mail: keary@scripps.edu

ORCID

Yang Gao: 0000-0001-9270-6251

Keary M. Engle: 0000-0003-2767-6556

\section{Notes}

The authors declare no competing financial interests.

\section{ACKNOWLEDGMENT}

This work was financially supported by the National Institutes of Health (5R35GM125052-03) and an ACS PRF Doctoral New Investigator Grant. Additionally, we gratefully acknowledge Tsinghua University Department of Chemistry for a Xuetang Program Scholarship (Z.-Q. W.). Dr. Jason Chen and Brittany Sanchez (Scripps Research Automated Synthesis Facility) are acknowledged for HRMS analysis. We further thank Dr. Milan Gembicky and Jake B. Bailey (UCSD) for X-ray crystallographic analysis.

\section{REFERENCES}

(1) (a) Miyaura, N.; Suzuki, A. Palladium-Catalyzed Cross-Coupling Reactions of Organoboron Compounds. Chem. Rev. 1995, 95, 2457-2483. (b) Lennox, A. J. J.; Lloyd-Jones, G. C. Selection of Boron Reagents for Suzuki-Miyaura Coupling. Chem. Soc. Rev. 2014, 43, 412-443. (c) Geier, S. J.; Westcott, S. Dehydrogenative Borylation: The Dark Horse in Metal-Catalyzed Hydroborations and Diborations? Rev. Inorg. Chem. 2015, 35, 69-79.

(2) (a) Shimizu, M.; Nakamaki, C.; Shimono, K.; Schelper, M.; Kurahashi, T.; Hiyama, T. Stereoselective Cross-Coupling Reaction of 1,1-Diboryl-1-alkenes with Electrophiles: A Highly Stereocontrolled Approach to 1,1,2-Triaryl-1-alkenes. J. Am. Chem. Soc. 2005, 127, 12506-12507. (b) Shimizu, M.; Shimono, K.; Schelper, M.; Hiyama, T. Stereoselective Cross-Coupling Reaction of 2,4-Diaryl1,1-diboryl-1,3-butadienes: Stereocontrolled Approach to 1,3,4,6-Tetraarylated 1,3,5-Hexatrienes Synlett 2007, 12, 1969-1971. (c) Wen, H.; Zhang, L.; Zhu, S.; Liu, G.; Huang, Z. Stereoselective Synthesis of Trisubstituted Alkenes via CobaltCatalyzed Double Dehydrogenative Borylations of 1-Alkenes. ACS Catal. 2017, 7, 6419-6425.

(3) (a) Hata, T.; Kitagawa, H.; Masai, H.; Kurahashi, T.; Shimizu, M.; Hiyama, T. Geminal Difunctionalization of Alkenylidene-Type Carbenoids by Using Interelement Compounds. Angew. Chem., Int. Ed. 2001, 40, 790-792. (b) Kurahashi, T.; Hata, T.; Masai, H.; Kitagawa, H.; Shimizu, M.; Hiyama, T. Geminal Dimetalation of Alkylidene-Type Carbenoids with Silylboranes and Diborons. Tetrahedron 2002, 58, 6381-6385. (c) Mkhalid, I. A. I.; Coapes, R. B.; Edes, S. N.; Coventry, D. N.; Souza, F. E. S.; Thomas, R. L.; Hall, J. J.; Bi, S.-W.; Lin, Z.; Marder, T. B. Rhodium Catalysed Dehydrogenative Borylation of Alkenes: Vinylboronates via C-H Activation. Dalton Trans. 2008, 8, 1055-1064. (d) Takaya, J.; Kirai, N.; Iwasawa, N. Efficient Synthesis of Diborylalkenes from Alkenes and Diboron by a New PSiP-Pincer Palladium-Catalyzed Dehydrogenative Borylation. J. Am. Chem. Soc. 2011, 133, 12980-12983. (e) Kirai, N.; Iguchi, S.; Ito, T.; Takaya, J.; Iwasawa, N. PSiP-Pincer Type Palladium-Catalyzed Dehydrogenative Borylation of Alkenes and 1,3-Dienes. Bull. Chem. Soc. Jpn. 2013, 86, 784-799. (f) Yoshii, D.; Jin, X.; Mizuno, N.; Yamaguchi, K. Selective 
Dehydrogenative Mono- or Diborylation of Styrenes by Supported Copper Catalysts. ACS Catal. 2019, 9, 3011-3016.

(4) (a) Morinaga, A.; Nagao, K.; Ohmiya, H.; Sawamura, M. Synthesis of 1,1Diborylalkenes through a Brønsted Base Catalyzed Reaction between Terminal Alkynes and Bis(pinacolato)diboron. Angew. Chem., Int. Ed. 2015, 54, 1585915862. (b) Krautwald, S.; Bezdek, M. J.; Chirik, P. J. Cobalt-Catalyzed 1,1-Diboration of Terminal Alkynes: Scope, Mechanism, and Synthetic Applications. J. Am. Chem. Soc. 2017, 139, 3868-3875. (c) Procter, R. J.; Uzelac, M.; Cid, J.; Rushworth, P. J.; Ingleson, M. J. Low-Coordinate NHC-Zinc Hydride Complexes Catalyze Alkyne C-H Borylation and Hydroboration Using Pinacolborane. ACS Catal. 2019, 9, 5760-5771.

(5) (a) Noguchi, H.; Hojo, K.; Suginome, M. Boron-Making Strategy for the Selective Synthesis of Oligoarenes via Iterative Suzuki-Miyaura Coupling. J. Am. Chem. Soc. 2007, 129, 758-759. (b) Noguchi, H.; Shioda, T.; Chou, C. M.; Suginome, M. Differentially Protected Benzenediboronic Acids: Divalent CrossCoupling Modules for the Efficient Synthesis of Boron-Substituted Oligoarenes. Org. Lett. 2008, 10, 377- 380. (c) Iwadate, N.; Suginome, M. Synthesis of Masked Haloareneboronic Acids via Iridium-Catalyzed Aromatic C-H Borylation with 1,8-Naphthalenediaminatoborane (danBH). J. Organomet. Chem. 2009, 694, 1713-1717. (d) Iwadate, N.; Suginome, M. Synthesis of B-Protected $\beta$-Styrylboronic Acids via Iridium-Catalyzed Hydroboration of Alkynes with 1,8Naphthalenediaminatoborane Leading to Iterative Synthesis of Oligo(phenylenevinylene)s. Org. Lett. 2009, 11, 1899-1902. (e) Iwadate, N.; Suginome, M. Differentially Protected Diboron for Regioselective Diboration of Alkynes: Internal-Selective Cross-Coupling of 1-Alkene-1,2-diboronic Acid Derivatives. J. Am. Chem. Soc. 2010, 132, 2548-2549. (f) Peng, S.; Liu, G.; Huang, Z. Mixed Diboration of Alkynes Catalyzed by LiOH: Regio- and Stereoselective Synthesis of cis1,2-Diborylalkenes. Org. Lett. 2018, 20, 7363-7366.

(6) (a) Yamamoto, K.; Mohara, Y.; Mutoh, Y.; Saito. S. Ruthenium-Catalyzed (Z)-Selective Hydroboration of Terminal Alkynes with Naphthalene-1,8-diaminatoborane. J. Am. Chem. Soc. 2019, 141, 17042-17047. (b) Yoshida, H.; Seki, M.; Kamio, S.; Tanaka, H.; Izumi, Y.; Li, J.; Osaka, I.; Abe, M.; Andoh, H.; Yajima, T.; Tani, T.; Tsuchimoto, T. Direct Suzuki-Miyaura Coupling with Naphthalene-1,8-diaminato (dan)-Substituted Organoborons. ACS Catal. 2020, 10, 346-351. (c) Mutoh, Y.; Yamamoto, K.; Saito, S. Suzuki-Miyaura CrossCoupling of 1,8-Diaminonaphthalene (dan)-Protected Arylboronic Acids. ACS Catal. 2020, 10, 352-357.

(7) Viereck, P.; Krautwald, S.; Pabst, T. P.; Chirik, P. J. A Boron Activating Effect Enables Cobalt-Catalyzed Asymmetric Hydrogenation of Sterically Hindered Alkenes. J. Am. Chem. Soc. 2020, 142, 3923-3930.

(8) (a) Lee, J. C.; McDonald, R.; Hall, D. G. Enantioselective Preparation and Chemoselective Cross-Coupling of 1,1-Diboron Compounds. Nat. Chem. 2011, 3, 894-899. (b) Feng, X.; Jeon, H.; Yun, J. Regio- and Enantioselective Copper(I)-Catalyzed Hydroboration of Borylalkenes: Asymmetric Synthesis of 1,1Diborylalkanes. Angew. Chem., Int. Ed. 2013, 52, 3989-3992.

(9) Liu, X.; Ming, W.; Luo, X.; Friedrich, A.; Maier, J.; Radius, U.; Santos, W.; Marder, T. Regio- and Stereoselective Synthesis of 1,1-Diborylalkenes via Brønsted Base-Catalyzed Mixed Diboration of Alkynyl Esters and Amides with BpinBdan. Eur. J. Org. Chem. 2020, 1-7.
(10) (a) Liu, Z.; Derosa, J.; Engle, K. M. Palladium(II)-Catalyzed Regioselective syn-Hydroarylation of Disubstituted Alkynes Using a Removable Directing Group. J. Am. Chem. Soc. 2016, 138, 13076-13081. (b) Derosa, J.; Cantu, A. L.; Boulous, M. N.; O’Duill, M. L.; Turnbull, J. L.; Liu, Z.; De La Torre, D. M.; Engle, K. M. Directed Palladium(II)-Catalyzed anti-Hydrochlorination of Unactivated Alkynes with HCl. J. Am. Chem. Soc. 2017, 139, 5183-5193. (c) Gao, D.-W.; Gao, Y.; Shao, H.; Qiao, T.-Z.; Wang, X.; Sanchez, B.; Chen, J. S.; Liu, P.; Engle, K. M. Cascade CuH-Catalysed Conversion of Alkynes into Enantioenriched 1,1-Disubstituted Products. Nat. Catal. 2020, 3, 23-29.

(11) (a) Romero, E. A.; Jazzar, R.; Bertrand, G. Copper-Catalyzed Dehydrogenative Borylation of Terminal Alkynes with Pinacolborane. Chem. Sci. 2017, 8 , 165-168 (b) Romero, E. A.; Jazzar, R.; Bertrand G. (CAAC)CuX-Catalyzed Hydroboration of Terminal Alkynes with Pinacolborane Directed by the X-Ligand. J. Organomet. Chem. 2017, 829,11-13.

(12) (a) Semba, K.; Fujihara, T.; Terao, J.; Tsuji, Y. Copper-Catalyzed Highly Regio- and Stereoselective Directed Hydroboration of Unsymmetrical Internal Alkynes: Controlling Regioselectivity by Choice of Catalytic Species. Chem. Eur. J. 2012, 18, 4179-4184. (b) Jang, W. J.; Lee, W. L.; Moon, J. H.; Lee, J. Y; Yun, J. Copper-Catalyzed trans-Hydroboration of Terminal Aryl Alkynes: Stereodivergent Synthesis of Alkenylboron Compounds. Org. Lett. 2016, 18, 1390-1393. (c) Hall, J. W.; Unson, D. M. L.; Brunel, P.; Collins, L. R.; Cybulski, M. K.; Mahon, M. F.; Whittlesey, M. K. Copper-NHC-Mediated Semihydrogenation and Hydroboration of Alkynes: Enhanced Catalytic Activity Using Ring-Expanded Carbenes. Organometallics 2018, 37, 3102-3110.

(13) (a) Liu, X.; Ming, W.; Zhang, Y.; Friedrich, A.; Marder, T. B. CopperCatalyzed Triboration: Straightforward, Atom-Economical Synthesis of 1,1,1Triborylalkanes from Terminal Alkynes and HBpin. Angew. Chem., Int. Ed. 2019, 58, 18923-18927. (b) Liu, X.; Ming, W.; Friedrich, A.; Kerner, F.; Marder, T. B. Copper-Catalyzed Triboration of Terminal Alkynes Using B2pin2: Efficient Synthesis of 1,1,2-Triborylalkenes. Angew. Chem., Int. Ed. 2020, 59, 304-309

(14) (a) Hammond, B.; Jardine, F. H.; Vohra, A. G. Carboxylatocopper(I) Complexes. J. Inorg. Nucl. Chem. 1971, 33, 1017-1024. (b) David. A.; Stefan, M.; Marcus, K.; Roy, B.; Tobias, R.; Steffen, S.; Lutz, M.; Michael, H.; Michael, M.; Heinrich, L. Copper(II) and Triphenylphosphine Copper(I) Ethylene Glycol Carboxylates: Synthesis, Characterisation and Copper Nanoparticle Generation. Dalton, Trans. 2013, 42, 15599-15609.

(15) (a) Buckley, B. R.; Dann, S. E.; Heaney, H.; Stubbs, E. C. Heterogeneous Catalytic Reactions "On Water" by Using Stable Polymeric Alkynylcopper(I) Pre-Catalysts: Alkyne/Azide Cycloaddition Reactions. Eur. J. Org. Chem. 2011, $770-776$.

(16) Xi, Y.; Hartwig, J. F. Mechanistic Studies of Copper-Catalyzed Asymmetric Hydroboration of Alkenes. J. Am. Chem. Soc. 2017, 139, 12758-12772

(17) Lipshutz, B. H.; Bošković, Ž. V.; Aue. D. H. Synthesis of Activated Alkenylboronates from Acetylenic Esters by $\mathrm{CuH}$-Catalyzed 1,2-Addition/Transmetalation. Angew. Chem., Int. Ed. 2008, 47, 10183-10186.

(18) In the 1,1-homodiboration reaction of phenylacetylene with HBdan, the dehydrogenative borylation cycle was found much faster than the hydroboration cycle. After 2 h, $50 \%$ of $3 \mathbf{a}$ and less than $5 \%$ of $\mathbf{4 a}$ were observed by ${ }^{1} \mathrm{H}$ NMR spectroscopy. 
TOC graphic

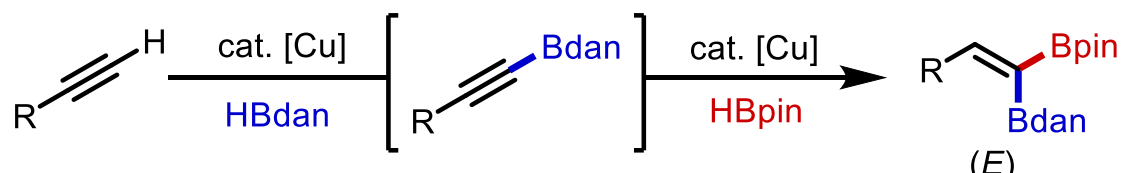

$(E)$

- exclusive E-selectivity - broad scope $\cdot$ mild conditions - sequential cross-couplings of the two $C-B$ bonds 